\title{
Editorial
}

\section{A Nobel prize to public science communication}

The Norwegian Nobel Committee has bestowed the 2007 Nobel Peace Price equally upon the scientists of the Intergovernmental Panel on Climate Change (IPCC) and Al Gore, former vice-President of the United States of America, with the same motivation: «for their efforts to build up and disseminate greater knowledge about man-made climate change, and to lay the foundations for the measures that are needed to counteract such change».

So, the IPCC and Al Gore have been awarded also and especially for their public science communication activity. It is a much more significant acknowledgement because it follows the award won by Al Gore at the 79th Academy Awards annual ceremony for his documentary An Inconvenient Truth.

The year about to end has seen a growing general concern on climate change - increasingly perceived as one of the most serious and urgent issues the entire humanity has to tackle - and this includes the crucial role public communication on the process has acquired in the solution to the problem. In fact, the 2007 Nobel Peace Prize and the 2007 Academy Award for Best Documentary Feature can be partially considered as awards to public science communication.

Certainly, science communication experts and stakeholders are well aware of the fact that communication to the general public performs a number of key functions in the search of a solution to technical-scientific issues. Which are also economic, social and political issues.

Through public communication, information reaches the whole range of people that either make relevant decisions (shareholders) or play, in any case, a relevant role in the process (stakeholders).

Besides, widespread communication contributes in particular to the education of the public opinion. In the case of global problems, such as climate change, it performs the crucial function of educating the world's public opinion which is the other global superpower - as somebody once said. The IPCC and Al Gore have played a fundamental role specifically in the construction of a global public opinion on climate change and it has been highly influential - for it has acted as a global superpower - on the decisions by the international community (for example, within the UN or the EU) and of single governments (a recent example is the change in the Australian government majority, caused also and especially by previous governmental choices on climate change, as many maintain).

Quite interestingly, the contribution to the construction of a global public opinion is twofold: it is implemented through explicit and rigorous forms of science communication (for example, the reports by IPCC) that reinforce the public understanding of climate change and through communication forms strongly featuring implicit messages (consider films about climate change, such as The Day After Tomorrow) that reinforce the public perception of climate change. Obviously, between these two extremes, there is a vast array of initiatives that stimulate both the public understanding and the public perception, of which the documentary by $\mathrm{Al}$ Gore is only the most notable instance.

However, it should be repeated, all of the experts are conscious of this. Now part of a widespread culture, public science communication has become a professional need for scientists and, at the same time, one of the pillars in the foundation to the modern law of citizenship in a democratic society.

The novelty - suggested by the Nobel Peace Prize awarded to the IPCC and Al Gore, besides the Oscar granted to Gore again - is that this awareness on the crucial role played by science communication is spilling from the experts' field and turning into a widespread awareness.

This does not only gratify the people like us who deal with public science communication in their profession. It also gives us a new responsibility. It pushes us to study more in depth our own activity, to experiment new communication forms, to give richer and more solid contents to the concept, still to be explored, of scientific citizenship.

Translated by Massimo Caregnato

Pietro Greco 